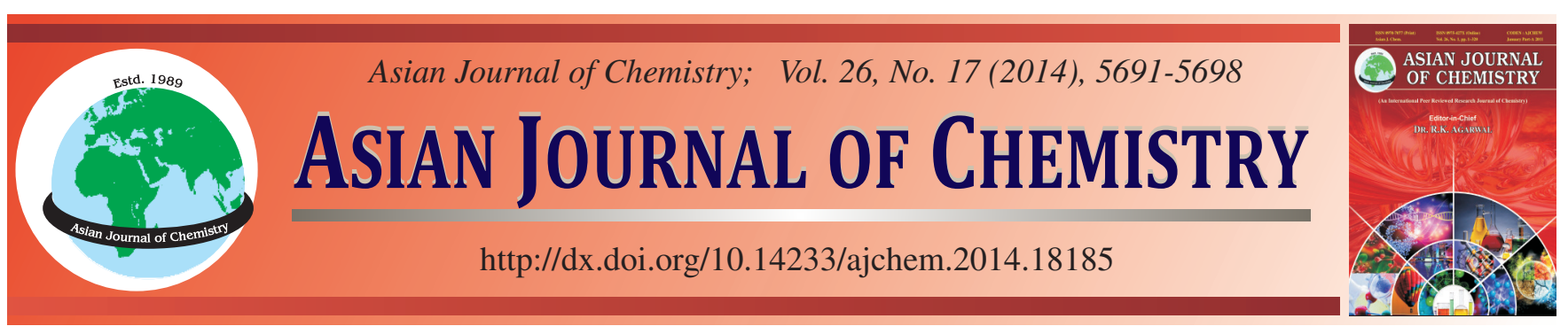

\title{
Application of Pressure-Sensitive Materials in Cement-Based Composites for Self-Assessment of Structural Degradation $\dagger$
}

\author{
AN CHENG
}

Department of Civil Engineering, National Ilan University, 1 Shen-Nong Road, Ilan 26047, Taiwan

Corresponding author: Fax: +886 3 9329286; Tel: +886 3 9317557; E-mail: ancheng@ niu.edu.tw

\begin{abstract}
The accumulation of internal defects in materials often results in degraded performance and the prevention of sudden material failure has become an important issue in materials research. In large structures such as buildings and bridges, sudden collapses due to the buildup of internal cracks can lead to significant losses, both in property and lives. If concrete structures were capable of performing self-assessments to reveal the presence of cracking, warnings could be issued before actual damage occurs. The addition of conductive materials is necessary to perform this kind of self-diagnosis in cement-based materials. This study added graphite to cement-based materials to investigate conductivity behavior and sensitivity to the effects of pressure. This study was conducted the mixture with a water-cementitious ratio of 0.6 for the preparation of cylindrical specimens $(\varphi 10 \mathrm{~cm} \times 20 \mathrm{~cm})$ in which 5,10 and $15 \%$ of the cement was replaced with graphite. We then investigated the influence of graphite contents on the electrical resistivity of the mortar and conducted cyclic loading tests to determine the relationship between rate of change in resistivity, stress and strain, and the application of load and the resulting changes in resistivity to indicate the existence and development of internal cracking. Results show that the addition of graphite to mortar significantly reduces the compressive strength of the concrete presented a linear decline proportional to the addition of graphite. With regard to resistivity, concrete with $5 \%$ graphite content reached the percolation threshold of the concrete and with an increase in the amount of graphite, resistivity gradually decreased. In cyclic loading tests with a fixed load, specimens with $5 \%$ or more graphite content were better able to reflect the relationship between loading and resistivity. The results of cyclic loading tests with loads of increasing and decreasing magnitude indicated that specimens with 10 and $15 \%$ graphite content provided a clearer indication of the relationship between loading and resistivity, due to their increased sensitivity to internal cracking conditions. In this study, the mixture in which $10 \%$ of the cement was replaced by graphite provided the best tradeoff between strength and sensitivity to the effects of cracking, making it the best option for practical use in construction.
\end{abstract}

Keywords: Graphite powder, Mortar, Resistivity change rate, Pressure sensitivity, Cyclic loading test.

ᄂ - - - - - - - - - - - - - - - - - - - - - - - - - - - -

\section{INTRODUCTION}

Concrete is currently the most widely applied material in building construction. It has been 190 years since Aspdin invented Portland cement and its low cost, high plasticity, easy obtainability and high compressive strength have led to its rapid and wide adoption. However, concrete has disadvantages including poor tensile strength, limited toughness and little resistance to bending, impact and explosions. This has led to the development of fiber-reinforced concrete, a new construction material with reduced brittleness. Generally, the electrical resistivity of concrete is high, ranging between $10^{4}$ and $10^{7} \Omega$, which necessitates the addition of materials such as graphite, carbon fibers, metal powder, or metal fibers to facilitate the conduction required for internal analysis. Smart materials must possess the mechanical properties and durability of traditional construction materials as well as the ability to imitate the sensory or control functions of biological organisms with regard to electrical, magnetic, thermal, optical, acoustic, chemical and rheological performance. The integration of smart materials represents the next important step in the development of materials. Hiarshi ${ }^{1}$ developed a self-repairing concrete by adding microencapsulated adhesives to cementbased materials. Dry ${ }^{2}$ also developed a self-repairing smart concrete with hollow glass fibers injected with a polymer solution to act as an adhesive. Currently, the primary functions of existing smart concretes include self-sensing, self-repair and self-regulation. This study used a pressure-sensitive smart material for the development of a model capable of diagnosing the extent of degradation in components. It is hoped that the 
achievements of this study will facilitate the development of smart construction materials in the future. Wittmann ${ }^{3}$ discovered that when a bar of cement paste was bent, electrodes attached to the opposite surfaces of the bar could detect voltage signals, indicating the presence of electromechanical activity within the material. When an electric current was passed through the bar, the bar bent, thereby proving the existence of the electromechanical effect. Shi and Chung ${ }^{4}$ observed variations in resistivity caused by tensile and compressive stress in cementbased composite materials containing short carbon fibers and continuous carbon fibers. It was also discovered that resistivity is correlated to the opening and closing of cracks within the material as well as the conditions of the cement-based material interface. Furthermore, reversible variations in resistivity were shown to be associated with elastic changes in the material. Similarly, irreversible changes in resistivity were shown to be associated with non-elastic deformations and fractures in the material. Thus, composite materials could be used as a sensitive indicator of pressure, tension and bending as well as the effects of static and dynamic loads. As such, the internal conditions of material could be used for non-destructive testing. Houssam et al. ${ }^{5}$ reported that adding carbon fibers to cement can increase the toughness and tensile and flexural strength of cement-based materials. Cao and Chung ${ }^{6}$ claimed that carbon fibers can enhance conductivity and pressure-sensitive properties of concrete. $\mathrm{Xu}$ and $\mathrm{Chung}^{7}$ found that ozone treatment can increase the adhesive strength between fibers and cement. Zheng and $\mathrm{Chung}^{8}$ examined the relationship between carbon fiber length and resistivity. Chung ${ }^{9}$ established that the optimal amount of carbon fiber in a concrete mix is less than $1.2 \%$ of the total volume. Concrete containing carbon fibers has been shown to possess self-diagnosing capabilities and is already being used in the construction of bridges and bank vaults. However, little research has been conducted to evaluate cement-based materials containing graphite. Previous workers ${ }^{8}$ studied the conductivity of cement-based materials with graphite and carbon fibers by measuring changes in the resistivity of materials due to damage. They also examined the influence of graphite and carbon fiber on the electromagnetic shielding properties of cement-based materials. Researchers have tended to focus on the pressure sensitivity of mortar rather than on cement-based materials. The addition of coarse aggregates increases the heterogeneity of composite materials, which also enhances the complexity of their conductive properties. Thus, this investigation of carbon fiber-reinforced cement-based materials is important to the further development of this technology. Fiber-reinforced concrete first appeared in 1910, when Chung ${ }^{9}$ introduced the concept of adding steel fibers to common concrete. After World War II, military engineering demanded that countries including Germany, Japan, France and the UK continue research on the addition steel fibers. Romualdi and Mandel ${ }^{10}$ made a breakthrough with a report on the mechanisms of crack development in steel fiber confinements. Based on the heterogeneity and anisotropy of fibers, fiber spacing theory was developed by Romualdi et al. ${ }^{11}$. However, linear elastic fracture mechanics is more widely accepted. When external force is applied, the presence of micro-cracks, voids and defects in concrete can lead to concentrations of stress, the extension and development of cracks and ultimately damage to the concrete. The incorporation of steel fibers in brittle base materials can prevent cracking and thus enhance the toughness of concrete. Technically, the proportion of steel fibers is proportional to toughness. Nonetheless, practical applications limit the amount of fibers to less than $2 \%$ of the overall volume, to prevent fiber segregation, ball formation and increased air volume.

The properties of carbon black vary according to the raw materials and the production method. The primary production method involves an oil furnace, the product of which comprises over $95 \%$ amorphous carbon nanoparticles. The production principle involves generating polycyclic aromatic hydrocarbons by placing hydrocarbons such as natural gas or petroleum in a high temperature environment. The polycyclic aromatic hydrocarbons then form carbon nuclei, which serve as the center around which particles gather to produce carbon black. The basic constituents of graphite are scaly flakes with a crystalline structure, comprising carbon atoms in a hexagonal arrangement. Carbon atoms in the same layer are covalently bonded, whereas different layers are connected by Van der Waals bonding. Conjugated double bonds between layers enable covalent $\pi$ electrons to move freely. Graphite is a stable mineral, which is resistant to strong acids and alkalis. Its crystal structures are smooth and lack functional groups, which prevent it from agglomerating easily. Although carbon black and graphite have the same composition, graphite possesses a higher density of $\pi$ electrons and superior conductivity. Graphite is also smooth and low in porosity and the lack of functional groups affects its affinity with other substances. Consequently, it disperses poorly in adhesives and does not easily form conductive pathways in coatings. In contrast, carbon black has rough surfaces, which enable it to disperse throughout adhesives and form conductive pathways, making it a better material for conductive coatings. Producing conductive fibers generally involves embedding or plating organic or inorganic conductive particles on the surface of fibers. This process is used in a wide range of applications, such as the elimination of static electricity, which is extremely important in the petroleum and electronics industries. Metal particles can be plated on fiber surfaces using chemical plating, or the fibers can be introduced into grooves of carbon powder, which adheres to the fibers. This is then placed in a heating tank to melt the fiber surfaces to embed the powder within the surfaces, resulting in conductive carbon fibers. The composite materials developed in these studies all provide a degree of conductivity, but each suffers from its own shortcomings. This study incorporated graphite into cement mortar and adopted the production method for slurry infiltrated fiber concrete (SIFCON) to produce graphite slurry infiltrated fiber cement-based material, a smart cement-based composite material with high conductivity.

\section{EXPERIMENTAL}

Cement: Type I Portland cement conforming to ASTM C150-09 was used in all mixes.

Fine aggregate: The fine aggregates had a fineness modulus of 2.71. The absorption of fine aggregates was $0.63 \%$, 
with a relative density of 2.59 under the saturated surface dry (SSD) condition

Graphite: The graphite powder used in this study was more than $99.9 \%$ pure, an average diameter of $5 \mu \mathrm{m}$.

Chemical admixture: To increase the fluidity of the formulas, we used high-performance, water-reducing Type $\mathrm{G}$ retarder, which has a water reduction rate of $12 \%$.

Conductive tape: The electrodes used in this study were made from double-sided conductive aluminum foil tape with a width of $2.5 \mathrm{~cm}$ and surface resistivity of $0.05 \mathrm{Ohms}$. The tape displayed good adhesiveness with the mortar specimens.

Test methods: In the mortar experiments, we studied the influence of graphite content on the workability of the mortar using water-cementitious ratios of 0.6 , varying the amount of cement replaced with graphite powder $(0,5,10$ and $15 \%)$. Appropriate quantities of water-reducing retarder were added to mixtures with fluidity below the requirements of $16 \mathrm{~cm}$. Using these mixtures, the details are presented in Table-1, the tests of mechanical properties were performed. The first letter $\mathrm{C}$ of the mixture numbers denotes the water-cementitiousitious ratios of 0.6. The $\mathrm{G}$ and the following number stand for the percentage of cement replaced with graphite. Therefore, replacement quantities of $0,5,10$ and $15 \%$ are denoted as G0, G5, G10 and G15. Table-1 shows the mix properties of mortar specimens.

The experiments in this study cast a total of 80 specimens made of 4 different mixes. All specimens were cured in saturated limewater until testing. For each mix, $\phi 100 \mathrm{~mm} \times 50 \mathrm{~mm}$ circular specimens were prepared to compressive strength and resistivity test.

Measurement of resistivity: The measurement of resistivity in conductive concrete requires electrodes, which can be embedded or taped to the surface of the material. For the sake of convenience, this study used conductive aluminum foil tape (width: $2.5 \mathrm{~cm}$ ) to create inexpensive electrodes. The most common methods for measuring resistivity include the two-electrode method and the four-electrode method. The fourelectrode method more effectively removes non-ohmic contact; therefore, we adopted this method. Fig. 1 displays the measurements of resistivity. We used a $7 \mathrm{~V}$ direct current power source with each specimen placed in series with a resistor of known resistance. As the current in the circuit is the same, V1/R1 = $\mathrm{V} 2 / \mathrm{R} 2$, where $\mathrm{R} 1$ and $\mathrm{R} 2$ are the resistance values of the specimen and the resistor and V1 and V2 were derived by data acquisition. In other words, R1 $=\mathrm{V} 1 / \mathrm{V} 2 \mathrm{R} 2$. After obtaining the base resistance values of the specimens, we were able to calculate the resistivity using the formula $\rho=\mathrm{RA} / \mathrm{L}$, where $\rho$ denotes resistivity $(\mathrm{k} \mathrm{Ohm} \mathrm{cm}), \mathrm{R}$ represents the resistance of the specimen (Ohm), A is the cross-sectional area of the specimen $\left(\mathrm{cm}^{2}\right)$ and $\mathrm{L}$ is the distance between the two electrodes (V1; cm) (Fig. 1).

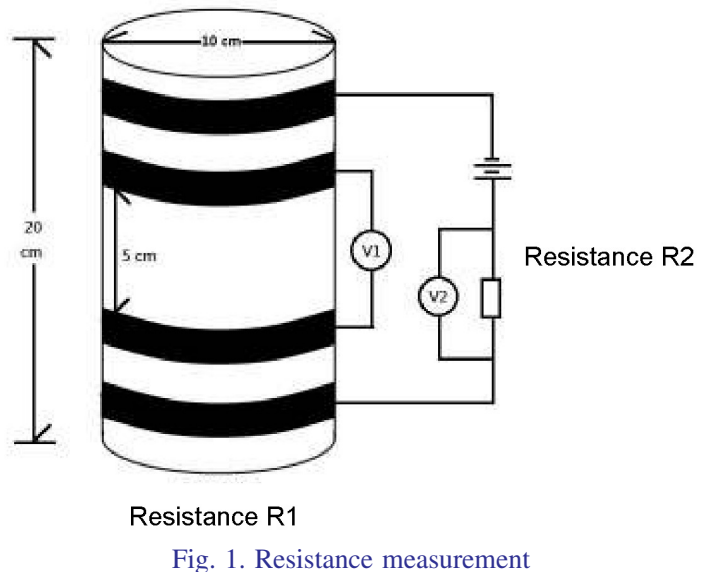

\section{RESULTS AND DISCUSSION}

Influence of graphite content on compressive strength and electrical resistivity of mortar: Table- 2 shows the compressive strength of the mixtures with various amounts of added graphite at 7 days, 28 days and 56 days. Table- 2 shows that the compressive strengths values exhibited by mixtures containing graphite were lower than those of the control group (no graphite), regardless of the water-cementitious ratio. From the perspective of strength reduction, replacing 5, 10 and $15 \%$ of the cement with graphite led to strength reductions of 15-20, 20-30 and 28-37\%, compared with the control group. These results demonstrate that the compressive strength of the specimens decreased with the amount of graphite added. This is likely due to the lubricity of the graphite surfaces in which the reduced friction between mortar particles was unable to resist the application of external force, thereby decreasing the compressive strength. The main flaw of graphite concrete is its low compressive strength. Therefore, the corresponding relationship between conductivity and strength must be considered when using graphite as a conductive material for smart concrete.

\begin{tabular}{cccc}
\multicolumn{5}{c}{ TABLE-2 } \\
\multicolumn{4}{c}{$\begin{array}{c}\text { COMPRESSIVE STRENGTH OF MIXTURES } \\
\text { AT DIFFERENT AGES (UNIT: MPa) }\end{array}$} \\
\cline { 2 - 4 } No. & 7 & Age (day) \\
\hline CG0 & 21.2 & 30.4 & 56 \\
CG5 & 18.1 & 24.7 & 29.0 \\
CG10 & 15.9 & 22.1 & 25.4 \\
CG15 & 16.0 & 19.2 & 24.4 \\
\hline
\end{tabular}

Influence of graphite content on electrical resistivity of mortar: Table-3 and Fig. 2 exhibit the resistivity coefficients measured using the direct current loop method. Table-3 exhibits only the results using the direct current loop method

\begin{tabular}{|c|c|c|c|c|c|c|c|}
\hline \multicolumn{8}{|c|}{$\begin{array}{c}\text { TABLE-1 } \\
\text { MIX PROPORTIONS }\left(\mathrm{kg} / \mathrm{m}^{3}\right)\end{array}$} \\
\hline Mix No. & $\mathrm{w}(\mathrm{cm})^{*}$ & Graphite content (wt. \%) & Water & Cement & Graphite & Fine aggregates & SP** \\
\hline CG0 & \multirow{4}{*}{0.6} & 0 & 300.7 & 501.2 & 0 & 1378.3 & 0 \\
\hline CG5 & & 5 & 299.5 & 474.2 & 25.0 & 1372.8 & 25.0 \\
\hline CG10 & & 10 & 298.3 & 447.5 & 49.7 & 1367.3 & 49.7 \\
\hline CG15 & & 15 & 294.6 & 420.9 & 74.3 & 1361.8 & 2.5 \\
\hline
\end{tabular}




\begin{tabular}{ccccc}
\hline \multicolumn{5}{c}{ TABLE-3 } \\
\multirow{2}{*}{$\begin{array}{c}\text { RESISTIVITY COEFFICIENTS MEASURED USING DIFECT } \\
\text { CURRENT LOOP METHOD (UNIT: K ohm cm) }\end{array}$} \\
\cline { 2 - 5 } No. & \multicolumn{4}{c}{ Age (day) } \\
\cline { 2 - 5 } & 7 & 14 & 28 & 56 \\
\hline CG0 & $4.8 \times 10^{3}$ & $4.65 \times 10^{3}$ & $3.51 \times 10^{3}$ & $3.23 \times 10^{3}$ \\
CG5 & $1.29 \times 10^{3}$ & $1.24 \times 10^{3}$ & $1.07 \times 10^{3}$ & $1.09 \times 10^{3}$ \\
CG10 & $7.52 \times 10^{2}$ & $7.43 \times 10^{2}$ & $6.59 \times 10^{2}$ & $6.43 \times 10^{2}$ \\
CG15 & $5.78 \times 10^{2}$ & $5.64 \times 10^{2}$ & $5.14 \times 10^{2}$ & $4.57 \times 10^{2}$ \\
\hline
\end{tabular}

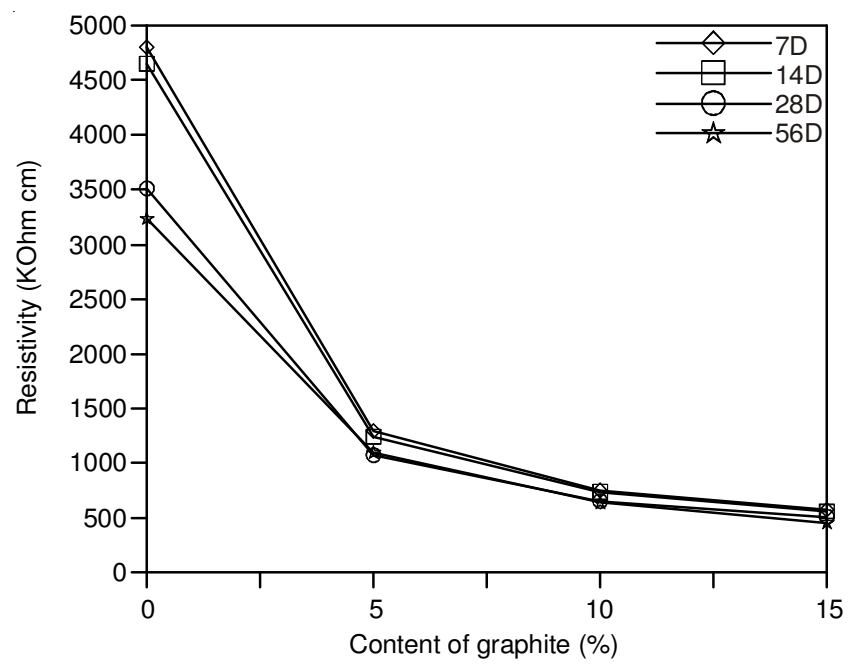

Fig. 2. Resistivity coefficients of specimens with $\mathrm{w} / \mathrm{cm}=0.6$ obtained using direct current loop method

from mixtures with a water-cementitious ratio of 0.6 . We selected this mixture because the drop in compressive strength with an increase in graphite content was less pronounced. The coefficient of resistivity in specimens with 5,10 and $15 \%$ graphite content were 70,83 and $87 \%$ lower than that of the control group, respectively.

The percolation threshold represents the point at which the addition of conductive media results in an abrupt drop in resistivity. Beyond this threshold, the decline in resistivity becomes moderate again. The above results demonstrate that even with graphite content of just $5 \%$, the percolation threshold of conductive media was surpassed. With a further increase in conductive media, resistivity began to decrease, but very slowly at first. In the above two experiments, the resistivity coefficients of the mixtures with $5 \%$ graphite content were more than $30 \%$ below those of the control group, indicating that $5 \%$ is already beyond the percolation threshold. Further comparison of mixtures with 10 and $15 \%$ graphite content revealed that the decline in resistivity had already decelerated. Thus, we were able to determine that a rough conductive network had formed in the mixture with $5 \%$ graphite content.

Relationships among resistivity changes, stress and strain: Figs. 3-7 present the changes in resistivity, corresponding to the stress-strain relationships derived from the compressive strength tests of mortar mixtures with four graphite contents. The $\mathrm{Y}$ axis indicates the difference between the obtained resistivity coefficient $(\mathrm{R})$ and the initial resistivity coefficient (R0) when divided by the initial resistivity coefficient in specimens under various loads. The strength values of CG0, CG5, CG10 and CG15 were 36.0, 29.5, 25.4 and 24.4 $\mathrm{MPa}$, respectively. As the load was increased, the regularity of

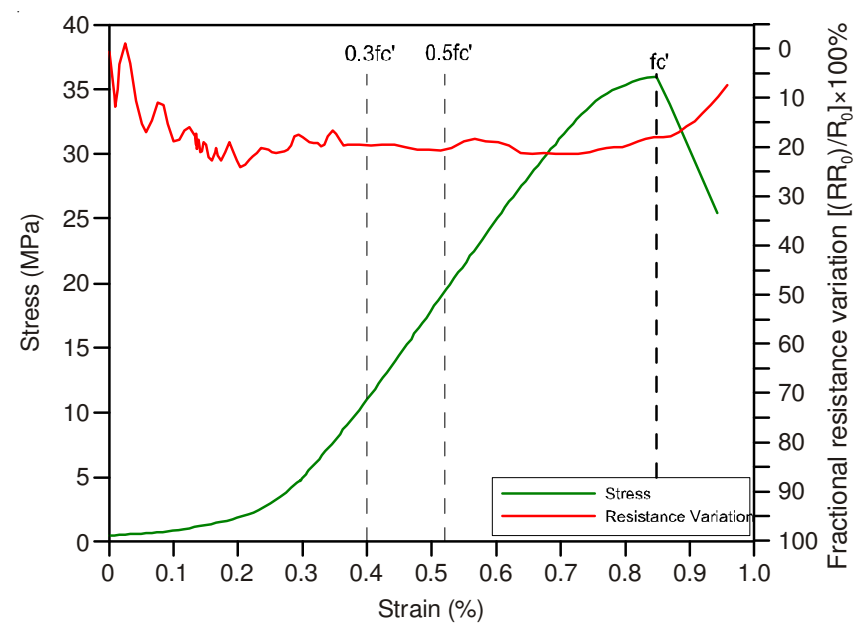

Fig. 3. Relationships among rate of change in resistivity, stress and strain (CG0)

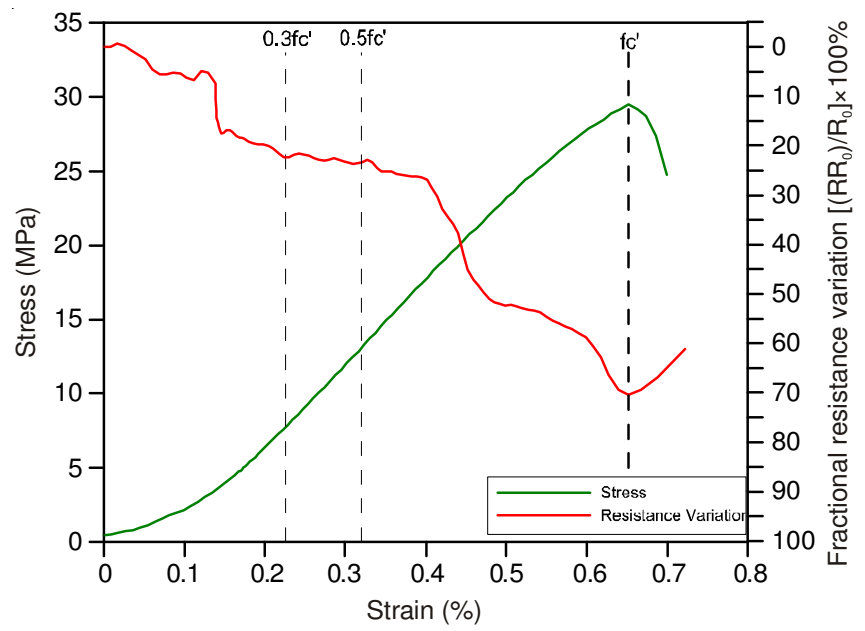

Fig. 4. Relationships among rate of change in resistivity, stress and strain (CG5)

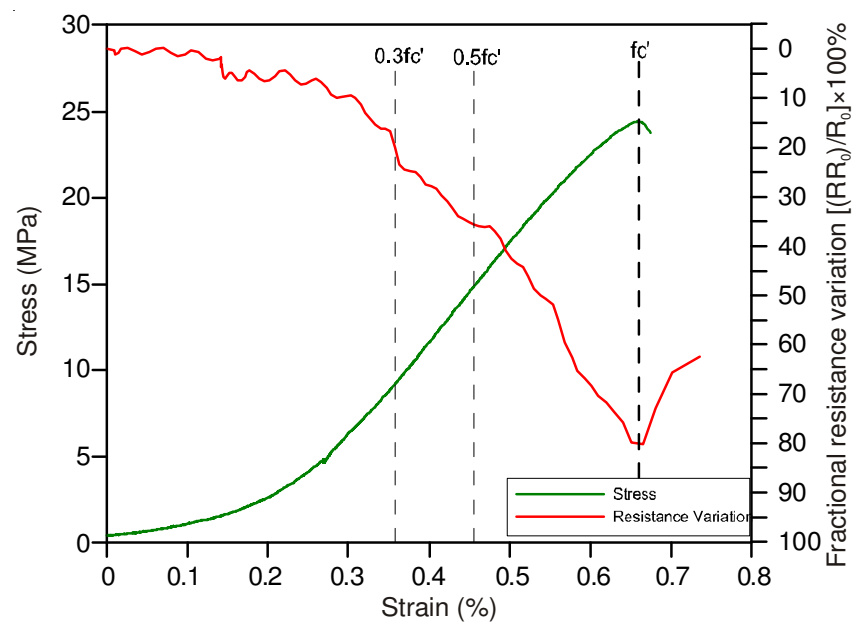

Fig. 5. Relationships among rate of change in resistivity, stress and strain (CG10)

specimen A (control group) was relatively poor. Early in the compression period, the coefficient of resistivity presented a linear decline in response to an increase in load. However, at a point between $50 \%$ of the maximum load and the rupture point, the resistivity coefficient fluctuated, such that we were unable to obtain an accurate indication of the internal damage 


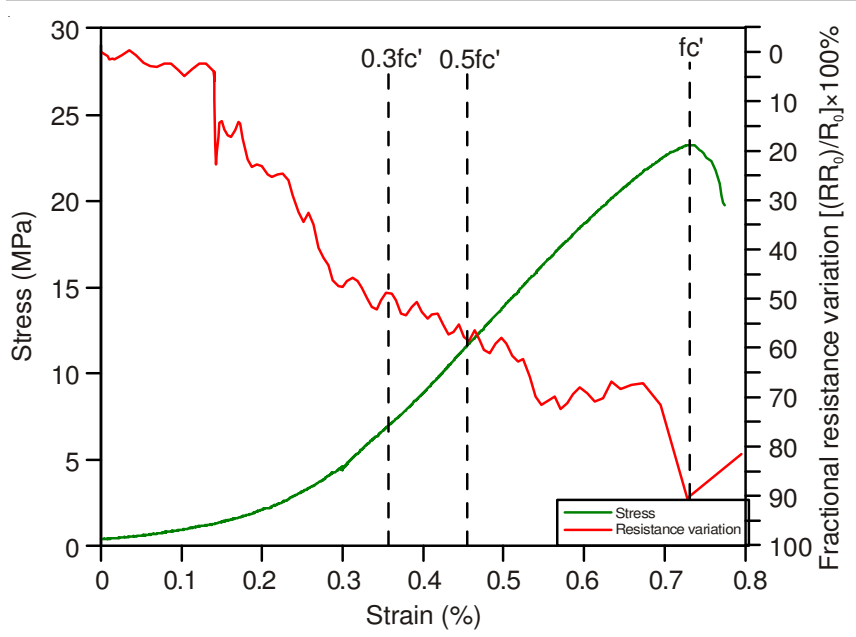

Fig. 6. Relationships among rate of change in resistivity, stress and strain (CG15)

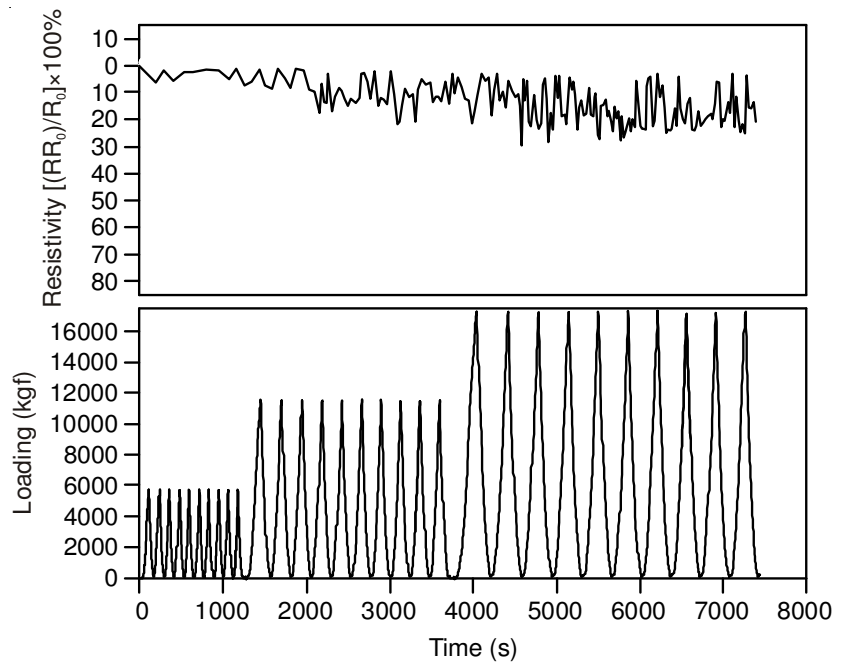

Fig. 7. Rate of change in resistivity of CG0 under cyclic loading (20-40$60 \%$ of the maximum strength)

of the specimen under pressure. The resistivity of specimen CG5 (5\% graphite content) also displayed a significant decreasing trend following an increase in load; under 30-50\% maximum load, the decline was more moderate. This continued to the point of rupture, where the decline in resistivity coefficient abruptly accelerated. This demonstrates that specimen B was unable to provide a comprehensive indication of the internal conditions of the specimen under lighter loads. The rate of change in the resistivity of specimen CG10 (10\% graphite content) and specimen CG15 (15\% graphite content) presented similar trends, decreasing with an increase in stress until rupture.

The greatest change in resistivity for specimen CG0 was about $30 \%$; for specimens CG5, CG10 and CG15 the rate of change was ca. 70, 80 and $90 \%$, respectively. This indicates that the compression of specimens containing graphite helps to establish conductive networks, which can lead to a more pronounced increase in resistivity.

The increase in resistivity in specimens CG5, CG10 and CG15 resulting from an increase in stress, can roughly be divided into three stages. The first stage presents fluctuations in the coefficient of resistivity at the initiation of compression. In the second stage, resistivity presents a stable decline as the load increases to a particular threshold. In the third stage, when the specimens approach the point of rupture, the coefficient of resistivity presents a sudden drop. Upon the rupture of the specimens, the growing number of cracks causes a significant and abrupt increase in resistivity. The three stages are at least partially correlated with the development of cracks in the mortar.

In summary of the above results, the addition of graphite powder to the specimens enhanced the relationship between the load applied in compressive strength tests and the rate at which resistivity in the material changed. Variations in the coefficient of resistivity in these mixtures were also more pronounced than those displayed by the control groups, thereby demonstrating that the addition of graphite can enhance the sensitivity of mortar to the effects of pressure.

Influence of graphite content on resistivity changes under the same load: The rate of change in the resistivity of various specimens undergoing the same load (maximum stress: $4.99 \mathrm{MPa}$ ) through 10 cycles. The graph for CG0 shows that after the first cycle, the coefficient of resistivity presented a decline that was clearly irreversible. This is because defects, pores and cracks become smaller with compression, resulting in irreversible deformations within the specimens. With further cycles, this decline is less pronounced. In samples without the addition of conductive media, changes in resistivity and loading appear irregular, due to a lack of sensitivity to the effects of pressure. In CG5, the coefficient of resistivity displayed reversible changes following the first cycle. In this case, a significant relationship was observed between loading and the rate at which resistivity changed, revealing that samples with $5 \%$ graphite content had already reached the percolation threshold resulting in the formation of a conductive network within the specimens, which enhanced their sensitivity to pressure. In CG10 and CG15, the changes in resistivity were also reversible. However, the coefficient of resistivity in specimens after unloading showed an increasing trend with the number of cycles. It is speculated that this is due to the accumulation of damage within the specimens after each cycle of loading and unloading.

The above results reveal that when cyclic loading was applied to mortar in the control group, no relationship was observed between the coefficient of resistivity and loading, indicating that sensitivity to the effects of pressure was poor. Replacing $5 \%$ of the cement with graphite powder led to the formation of a basic conductive network, such that all specimens at or above this content of graphite presented a significant relationship between the coefficient of resistivity and loading. Thus, it is concluded that the addition of graphite powder enhances sensitivity to the effects of pressure. In specimens with 10 and $15 \%$ graphite content, the coefficient of resistivity increased with the number of cycles. It is speculated that this is due to the reduced compressive strength in these two specimens (the specimens with 10 and $15 \%$ graphite contents were loaded with 20 and $22 \%$ of the maximum strength). Under a fixed load, the damage occurring within the specimens resulted in an irreversible increase in resistivity. In addition, the specimens with 10 and $15 \%$ graphite content were clearly more susceptible to cracking. We therefore applied 10 cycles 
of loading at 20,40 and $60 \%$ of the maximum strength values, to observe trends in resistivity and their relationships with cracking.

Rate of change in resistivity under cyclic loading: Previous studies ${ }^{12}$ have indicated that fine cracks exist in most concrete; however, stress resulting from loading at between 0 and $30 \%$ of the maximum load remains within the elastic stage and therefore does not promote the development of the fine cracks. In contrast, loadings exceeding 30-40\% of the maximum strength inevitably leads to the growth of fine cracks within the concrete. Specimens subjected to loading equal to $70 \%$ of its maximum strength will create visible cracks within the concrete and on the surface, which increase until rupture.

To simulate some of the loading processes that may occur, we conducted cyclic loading tests with a variety of loads, equivalent in magnitude to 20,40 and $60 \%$ of the maximum loads for each of the specimens in the tests. These loads were applied in two orders: $20-40-60 \%$ (Order A) and 60-40-20 \% (Order B). This helped to elucidate how the changes in resistivity occurred when specimens underwent compression of increasing magnitude and when specimens underwent compression of decreasing magnitude. Using Order A, loading a $20 \%$ reveals the relationship between resistivity and loading when the mortar remains in the elastic stage. Loading of $40 \%$ indicates the relationship between changes in resistivity and sensitivity to pressure. The final $60 \%$ loading reveals the relationship between changes in resistivity and pressure in specimen approaching $70 \%$ of their maximum strength. We opted not to apply loadings equal to $70 \%$ of the maximum strength because all specimens tend to form visible cracks under such pressures. Following the appearance of visible cracks, the issue of sensitivity to pressure is moot, with regard to the rate of crack generation. Using Order B, we were able to observe the relationship between sensitivity to pressure and the rate at which resistivity changes as specimens are first subjected to strong compressive forces followed by forces of decreasing magnitude. This also made it possible to observe whether initial internal cracking continues to develop, even when stress levels are reduced.

Figs. 7-10 display the results from Order A (20-40-60\%). As can be seen, under the $20 \%$ loading, changes in resistivity in the control group were relatively small (5\%) and no significant relationship was observed between these values and application of loading. When the magnitude of loading was increases to 40 and $60 \%$ of the maximum strength, variations in resistivity increased noticeably (approximately between 15 and $30 \%$ ). However, no corresponding relationship was observed between resistivity and loading. In the specimen with $5 \%$ graphite content, loading of $20 \%$ altered resistivity by $15 \%$, which is higher than that observed in the control group and resistivity measurements presented a clear correspondence to cyclic changes in loading; i.e., increases in cyclic load led to a pronounced increase in the change in resistivity and a clear relationship with the cycles of applied stress. This is because the magnitude of the applied load is proportional to the damage inflicted on the mortar specimens. The application of high loads produces a number of cracks that heal under loading but revert after unloading, thereby increasing resistivity. Higher loads promoted greater variations in resistivity

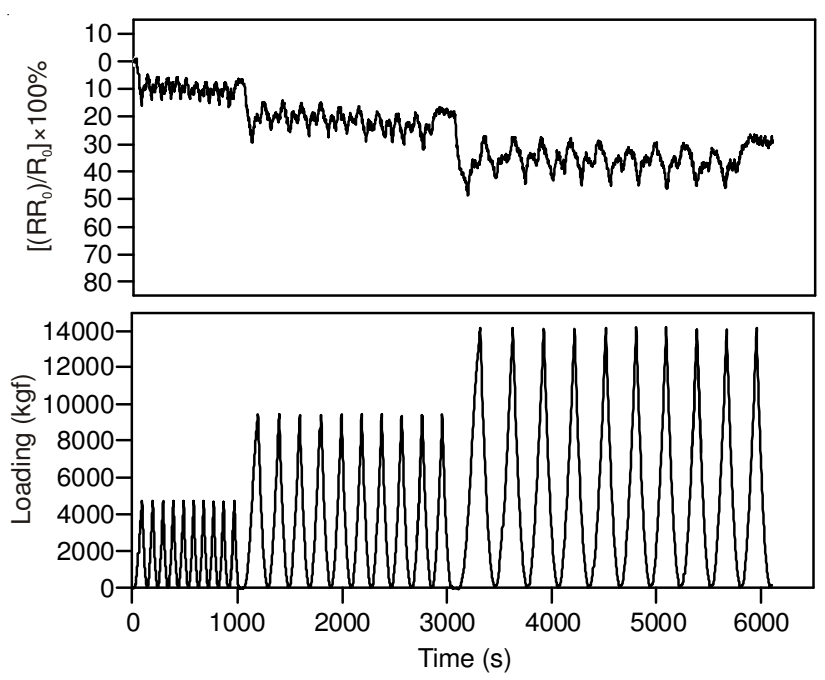

Fig. 8. Rate of change in resistivity of CG5 under cyclic loading (20-40$60 \%$ of the maximum strength)

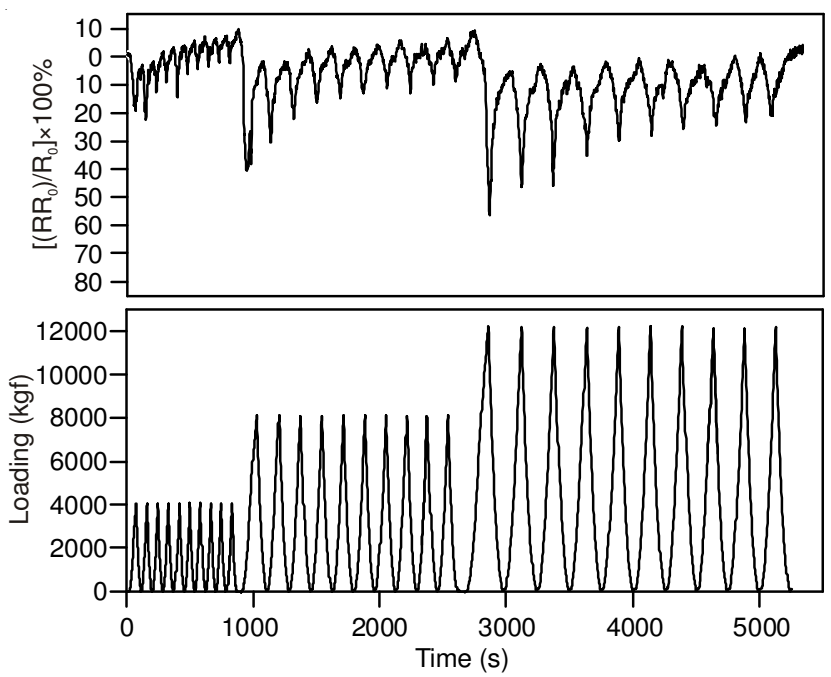

Fig. 9. Rate of change in resistivity of CG10 under cyclic loading (20-40$60 \%$ of the maximum strength)

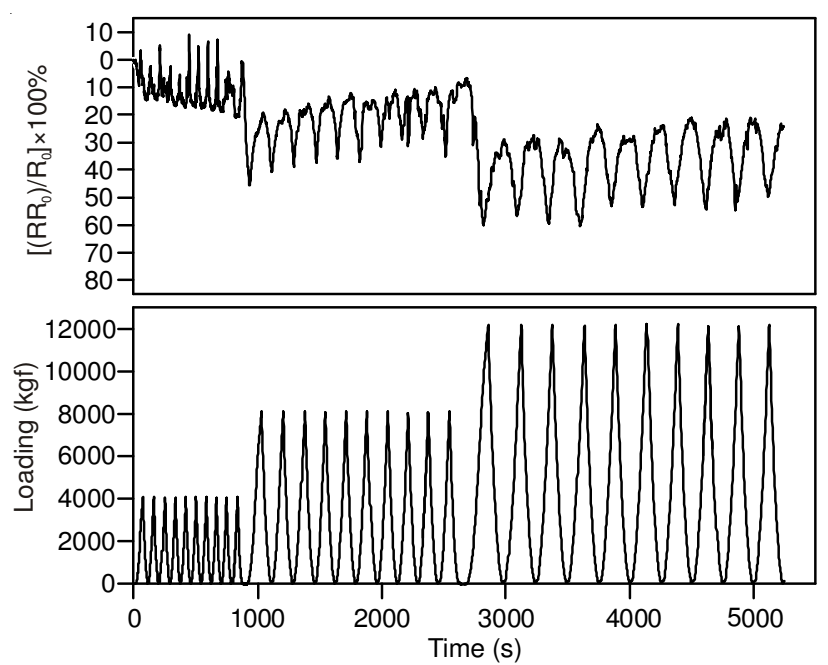

Fig. 10. Rate of change in resistivity of CG15 under cyclic loading (20-40$60 \%$ of the maximum strength)

and changed cyclically with the loading. The specimens with 10 and $15 \%$ graphite content were similar to the specimen 
with $5 \%$ graphite content, considering the fact that they all presented clear relationships between resistivity and loading. One difference however was that the coefficient of resistivity in specimens with 10 and $15 \%$ graphite content gradually increased with each cycle of maximum loading and unloading. It is speculated that this is due to the accumulation of damage in the specimens with each application of stress. As the load was increased, the formation of micro-cracks damaged the conductive network within the specimen, resulting in an irreversible increase in resistivity. Mindess et al. ${ }^{12}$ indicated that under loadings equal to $30-40 \%$ of the maximum strength, the formation of micro-cracks is consistent with the increased resistivity in specimens containing 10 and $15 \%$ graphite under cyclic loads of 40 and $60 \%$ of the maximum strength. Nevertheless, the two specimens began to show irreversible increases in resistivity under cyclic loads equal to $20 \%$ of the maximum strength. The may have been caused by the graphite powder acting as a filler material in the mortar. The saturated water absorption results indicate that higher graphite content led to more pronounced porosity in later ages than in earlier ages. This is a clear indication that the graphite was hindered in its hydration reaction with the cement. Furthermore, the smooth surfaces of the graphite resulted in poorer adhesion with the other constituents of the mortar. This may explain the formation of micro-cracks and irreversible increases in resistivity in the specimens, even under loadings of only $20 \%$, which is inconsistent with the theory proposed in previous research ${ }^{12}$.

Figs. 11-14 present the results of Order B (60-40-20\%). As can be seen, the variations in resistivity in the control group appear irregular and indicate no corresponding relationship with the applied stress. In contrast, specimens containing graphite clearly present this relationship, revealing an irreversible increase in resistivity with the number of cycles. In the specimen with $5 \%$ graphite content, the rate of change in resistivity increased from $-10 \%$ following the first unloading (60\% of the maximum strength) to approximately $0 \%$ following unloading at the end of the test $(20 \%$ of the maximum strength). In specimens with $10 \%$ and $15 \%$ graphite content, the rate of change in resistivity increased from -7 , $-15 \%$ to $5 \%$ and $10 \%$, respectively. This was due to the damage inflicted on the specimens after the application of a $60 \%$ load, which created numerous cracks, damaged the internal conductive network and led to a subsequent increase in resistivity with all subsequent applications of load. Subsequent loadings at 40 and $20 \%$ of the maximum strength did not produce as much stress as the initial load of $60 \%$; therefore, the specimen was unable to become any more compact as to reduce the resistivity. Due to the numerous cracks already existing in the specimens, even small amounts of stress were sufficient to increase cracking and resistivity. The above results demonstrate that the specimen with $15 \%$ graphite content gives a clear indication of the amount of cracking in the specimens. The specimens with 5 and $10 \%$ graphite content presented the increase in resistivity less clearly. However, the compressive strength of these samples was higher than that of the specimen with $15 \%$ graphite content. Thus, these mixtures could be considered options for construction applications requiring higher strength characteristics.

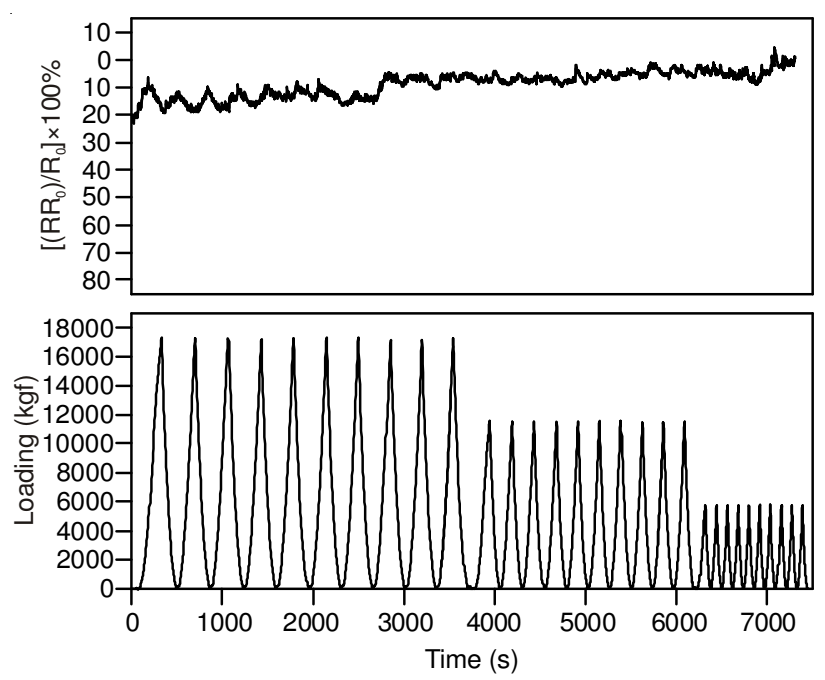

Fig. 11. Rate of change in resistivity of CG0 under cyclic loading (60-40$20 \%$ of the maximum strength)

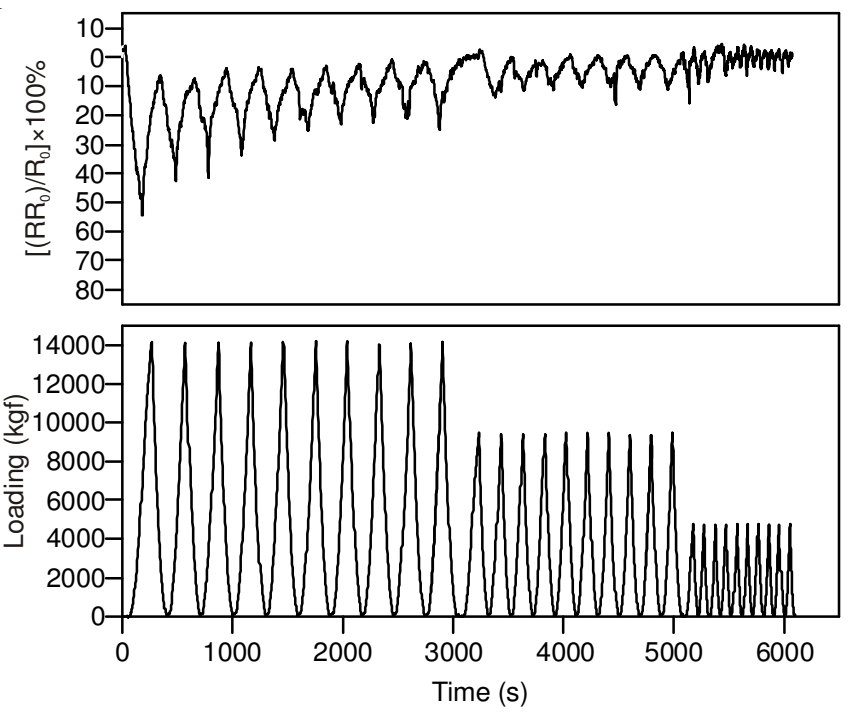

Fig. 12. Rate of change in resistivity of CG5 under cyclic loading (60-40$20 \%$ of the maximum strength)

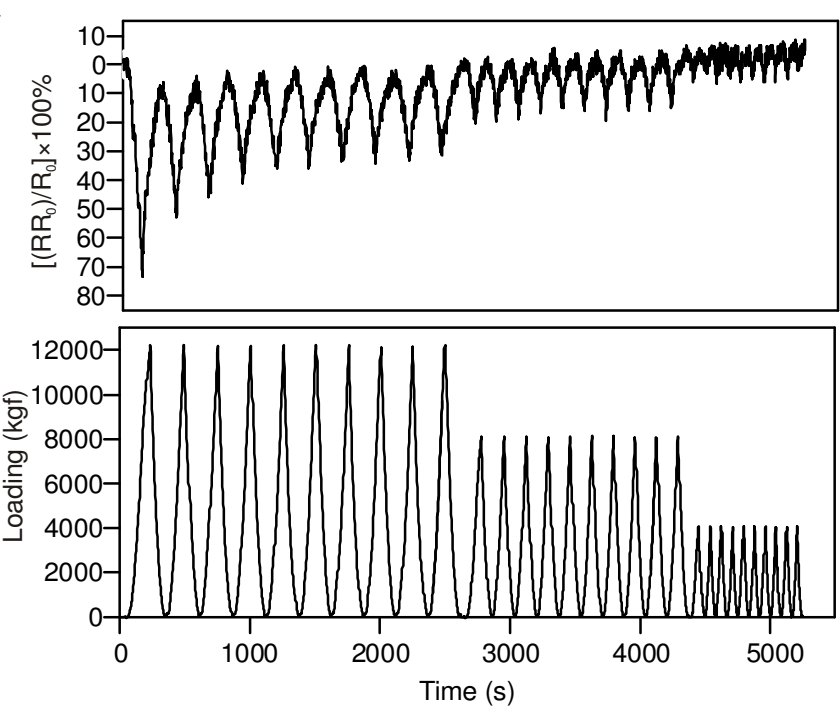

Fig. 13. Rate of change in resistivity of CG10 under cyclic loading (60-40$20 \%$ of the maximum strength) 


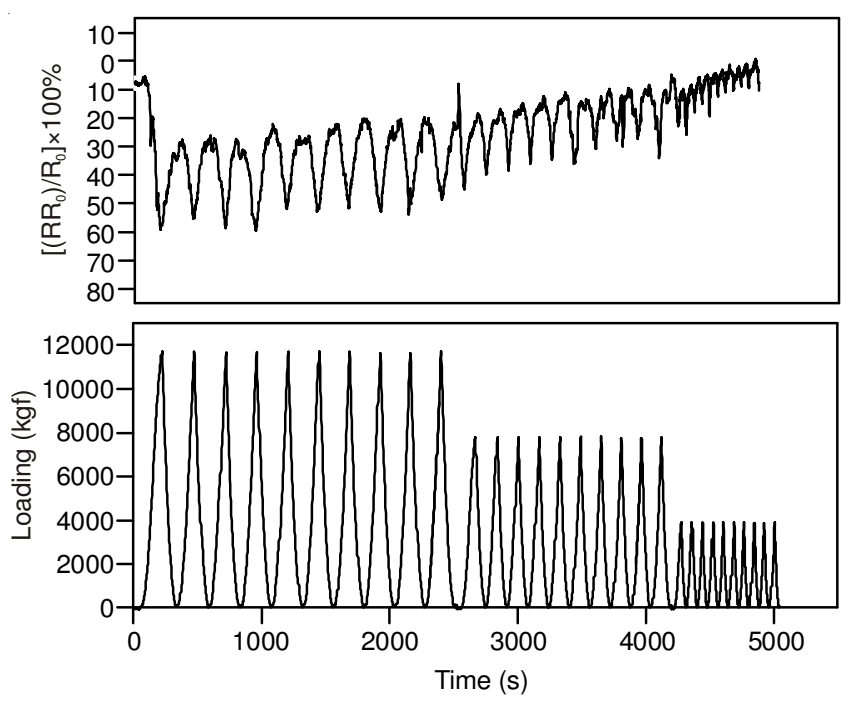

Fig. 14. Rate of change in resistivity of CG15 under cyclic loading (60-40$20 \%$ of the maximum strength)

\section{Conclusion}

This study replaced various proportions of the cement in mortar with graphite powder and investigated the mechanical properties and sensitivity to the effects of applied pressure in the resulting samples. Based on these results, we derived the following conclusions:

- The addition of graphite powder decreases the compressive strength of the mortar, which presents a linear decline with an increase in graphite powder content. This can be attributed to the smoothness of the graphite surfaces, which degrades adhesiveness within the mortar, such that the resulting samples are more prone to damage under the application of stress.

- Mortar mixtures in which 5, 10 and $15 \%$ of the cement was replaced with graphite displayed clear relationships between resistivity and the load applied in compression tests. These results also indicate that changes in resistivity became increasingly pronounced with graphite content, indicating that graphite powder can be used to enhance the sensitivity of mortar to the effects of pressure.
- In cyclic loading tests with a fixed load, the specimens with $5 \%$ or more graphite content provided a clear indication of the relationships between changes in resistivity and applied load. The changes in resistivity in the specimens with 10 and $15 \%$ graphite content also increased with the number of cycles, thereby demonstrating that these two mixtures were highly sensitivity to cracking.

- Cyclic loading tests using loads of 20-40-60\% presented results similar to those of the cyclic tests with a fixed load. Under less compression, specimens with 10 and $15 \%$ graphite content presented an increase in resistivity with the number of cycles, such that the change in resistivity could be used as a proxy to estimate the rate of crack generation. The rate of change in the resistivity of specimens with $5 \%$ graphite content did not present evidence of cracking until the application loads equal to $60 \%$ of the maximum strength. Clearly, the specimens with 10 and $15 \%$ graphite content have greater sensitivity to the effects of pressure.

- In cyclic loading tests involving loads of 60-40-20\%, the specimen with $15 \%$ graphite content presented a clearer indication as to the extent of internal cracking under smaller loads following the initial larger loads.

The above findings show that the mixture in which $10 \%$ of the cement was replaced by graphite provided the best tradeoff between strength and sensitivity to the effects of cracking, making it the best option for practical use in construction.

\section{REFERENCES}

1. H. Hiraishi, Concrete J., 36, 11 (1998).

2. C. Dry, Smart Mater. Struct., 3, 118 (1994).

3. F.H. Wittmann, Cement Concr. Res., 3, 601 (1973).

4. Z.-Q. Shi and D.D.L. Chung, Cement Concr. Res., 29, 435 (1999).

5. H.A. Toutanji, T. El-Korchi and R.N. Katz, Cement Concr. Comp., 16, 15 (1994).

6. J.Y. Cao and D.D.L. Chung, Cement Concr. Res., 31, 1519 (2001)

7. Y.S. Xu and D.D.L. Chung, ACI Mater. J., 97, 333 (2000).

8. Q. Zheng and D.D.L. Chung, Cement Concr. Res., 19, 25 (1989).

9. D.D.L. Chung, Composites Part B, 31, 511 (2000).

10. J.P. Romualdi and J.A. Mandel, J. Am. Concr. Inst., 61, 657 (1964).

11. J.P. Romualdi, in eds.: A.E. Brooks and K. Newman, The Structure of Concrete, Proceedings of Int. Conf., London, pp. 190-250 (1968).

12. S. Mindess, J.F. Young and D. Darwi,, Concrete, Prentice Hall, edn 2 (1996). 